\title{
Prevalence and correlates of depressive symptoms among Chinese patients with cataracts treated in tertiary general hospitals
}

\author{
Zhong-Hua Liu ${ }^{1}$, Chang-Zheng Chen ${ }^{2}$, Cong Gao ${ }^{1}$, De-Yi Zhou ${ }^{\text {Corresp. } 3}$ \\ 1 Department of Ophthalmology, Wuhan Hankou Hospital, Wuhan, China \\ 2 Department of Ophthalmology, Renmin Hospital of Wuhan University, Wuhan, China \\ 3 Department of Psychiatry, The Affiliated Wuxi Mental Health Center of Nanjing Medical University, Wuxi, China \\ Corresponding Author: De-Yi Zhou \\ Email address: 397556051@qq.com
}

Background. Previous studies recruited unrepresentative samples of Chinese patients with cataract and reported a wide range of prevalence of depressive symptoms in this patient population (18.0-89.7\%). The present study determined the prevalence and correlates of depressive symptoms among a consecutive sample of Chinese patients with cataract treated in tertiary general hospitals.

Methods. A total of 339 patients with cataract were consecutively selected from ophthalmology departments of two large general hospitals in Wuhan, China. Depressive symptoms were assessed with the Chinese Hospital Anxiety and Depression Scale. Logistic regression was used to identify factors that were associated with depression.

Results. The prevalence of depressive symptoms was 23.9\% (95\%Cl: 19.4-28.4\%) among patients with cataract. Correlates for depressive symptoms include an education level of primary school and below $(\mathrm{OR}=1.93, \mathrm{P}=0.038)$, marital status of "others" $(\mathrm{OR}=3.15, \mathrm{P}<0.001)$, poor family economic status $(\mathrm{OR}=2.26, \mathrm{P}=0.010)$, nuclear cataract $(\mathrm{OR}=4.32, \mathrm{P}<0.001)$, and mixed cataract $(\mathrm{OR}=2.76, \mathrm{P}=0.017)$.

Conclusions. Depressive symptoms are common among Chinese patients with cataract treated in large general hospitals. Patients who are poorly educated, have a marital status other than "married", have poor family economic status, and suffer from nuclear and mixed cataracts are at greater risk for depressive symptoms. 


\section{Prevalence and correlates of depressive symptoms}

3 among Chinese patients with cataracts treated in

\section{4 tertiary general hospitals}

5

6

7

8

Zhong-Hua Liu ${ }^{1}$, Chang-Zheng Chen ${ }^{2}$, Cong Gao ${ }^{1}$, De-Yi Zhou ${ }^{3 *}$

${ }^{1}$ Department of Ophthalmology, Wuhan Hankou Hospital, Wuhan, Hubei Province, China;

${ }^{2}$ Department of Ophthalmology, Renmin Hospital of Wuhan University, Wuhan, Hubei Province, China;

${ }^{3}$ Department of Psychiatry, The Affiliated Wuxi Mental Health Center of Nanjing Medical University, Wuxi, Jiangsu Province, China.

Corresponding Author:

De-Yi Zhou ${ }^{3}$

Wuxi Mental Health Center, No. 156, Qianrong Road, Binhu District, Wuxi, Jiangsu Province, China.

Email address: 397556051@qq.com

\section{Abstract}

Background. Previous studies recruited unrepresentative samples of Chinese patients with cataract and reported a wide range of prevalence of depressive symptoms in this patient population (18.0-89.7\%). The present study determined the prevalence and correlates of depressive symptoms among a consecutive sample of Chinese patients with cataract treated in tertiary general hospitals.

Methods. A total of 339 patients with cataract were consecutively selected from ophthalmology departments of two large general hospitals in Wuhan, China. Depressive symptoms were assessed with the Chinese Hospital Anxiety and Depression Scale. Logistic regression was used to identify factors that were associated with depression. 
31 Results. The prevalence of depressive symptoms was 23.9\% (95\%CI: 19.4-28.4\%) among

32 patients with cataract. Correlates for depressive symptoms include an education level of primary

33 school and below $(\mathrm{OR}=1.93, \mathrm{P}=0.038)$, marital status of "others" $(\mathrm{OR}=3.15, \mathrm{P}<0.001)$, poor

34 family economic status $(\mathrm{OR}=2.26, \mathrm{P}=0.010)$, nuclear cataract $(\mathrm{OR}=4.32, \mathrm{P}<0.001)$, and mixed

35 cataract $(\mathrm{OR}=2.76, \mathrm{P}=0.017)$.

36 Conclusions. Depressive symptoms are common among Chinese patients with cataract treated in

37 large general hospitals. Patients who are poorly educated, have a marital status other than

38 "married", have poor family economic status, and suffer from nuclear and mixed cataracts are at 39 greater risk for depressive symptoms.

40

\section{Introduction}

42 In China, cataract is the top leading cause of blindness and low vision among middle-aged and older adults, and an estimated over one-third of this population suffer from cataract (Tang et al. 2016; Tian et al. 2014; Yuan \& Li 2016). In addition to the severe functional impairments and disability due to cataract, cataract has been associated with increased risk of mental health problems such as depression and mental health services utilization (Meuleners et al. 2013). The comorbid depression further exacerbates the poor cognitive and physical health, delays recovery from surgery, and increases mortality in people with cataract (Pellegrini et al. 2020). Therefore, a greater understanding on the clinical epidemiology of depression in this patient population may help the early identification of those at risk for depression and improve their mental and physical wellbeing.

In the literature, there have been some studies investigating depression in patients with cataract (Fraser et al. 2013; Mitsonis et al. 2006; Mylona et al. 2020). For example, in Australia and Canada, researchers used Geriatric Depression Scale (GDS) to assess the severity of depressive symptoms in 329 and 672 patients awaiting cataract surgery, respectively. The two studies reported similarly high prevalence of clinically significant depressive symptoms $(28.9 \%$ and 26.0\%) and identified a few correlates of depression such as poor visual acuity and major medical conditions (Freeman et al. 2009; Palagyi et al. 2016). In China, to the best of our known, four studies have investigated the prevalence of depression in patients with cataract (Chen et al. 2019; Chen 2010; He \& Gao 2015; Wang et al. 2016; Zhang et al. 2018). These studies assessed depressive symptoms with a variety of self-report depression scales (i.e., GDS, Zung's Self- 
62 rating Depression scale [SDS], 9-item Patient Health Questionnaire[PHQ-9], and Hospital

63 Anxiety and Depression Scale [HADS]) and reported a wide range of prevalence rates: 18.0-

64

65

66

67

68

69

70

71

72

73

74

75

76

77

78

79

80

81

82

83

84

85

86

87

88

89

90

91

92

89.7\%. SDS and PHQ-9, although both are reliable for measuring depressive symptoms in the general population, they are not able to accurately evaluate depressive symptoms among patients with cataract, because they have items of somatic symptoms, which are related to aging itself and very common among patients with chronic illnesses. Due to this, there may be false-positive cases with depressive symptoms in prior studies with samples of Chinese patients with cataract. Therefore, existing studies using SDS and PHQ-9 may have overestimated the prevalence of depressive symptoms among Chinese patients with cataract. Further, these prior studies often recruited small and convenient samples and seldom considered clinical factors associated with depression in Chinese individuals with cataract.

Given the clinical importance of comorbid depression in patients with cataract and limitations in previous Chinese studies, the present study assessed the prevalence of depressive symptoms and their associated factors in a consecutive clinical sample of Chinese patients with cataract treated in tertiary general hospitals.

\section{Materials \& Methods}

\section{Participants}

This cross-sectional study was conducted from March to July, 2019. Participants were adults receiving treatment in outpatient and inpatient departments of ophthalmology at Wuhan Hankou Hospital and Renmin Hospital of Wuhan University, two large tertiary general hospitals in Wuhan, China. By using consecutive sampling method, patients who were 18 years and older, met the diagnostic criteria for cataract after slit-lamp examination, and were capable of communicating with investigators were all invited to participate in the study. We excluded patients with eye diseases other than cataract, dementia, brain organic mental disorders, and psychotic disorders, as well as those who were too physically ill to complete the interview. In our pilot study with a small sample of 20 patients with cataract, the prevalence of depressive symptoms was $25 \%$. Parameters for sample size estimation of our study were: a prevalence rate of $25 \%$, a precision of 0.05 , a response rate of 0.9 , and a confidence interval of 95\% (95\%CI). PASS 2005 (LLC, Kaysville, UT, USA) estimated that a minimum sample size of 316 would ensure the stability of our prevalence estimate. 
93

94

95 96

97

98

99

100

101

102

103

104

105

106

107

108

109

110

111

112

113

114

115

116

117

118

119

120

121

122

123

Written informed consent was obtained from patients and their caregivers and declarations of anonymity and confidentiality had been made before the start of data collection. The Ethics Committee of Wuhan Hankou Hospital approved the study protocol (approval number: 2019-SRY0036).

\section{Procedures and instruments}

Data were collected by using a standardized questionnaire. Because most patients had difficulties in reading due to visual acuity problems, the questionnaire was completed in a face-to-face and one-to-one interview manner. The study investigators were four trained master students in clinical ophthalmology. Before the formal study, 15 patients with cataract were recruited to participate in the training sessions for the investigators. The inter-rater reliability of the four investigators of the HADS depression score was satisfactory (intra-class correlation coefficient $=0.92)$.

Socio-demographic variables in the questionnaire were gender, age, education, marital status, current residence place (urban, rural, and urban-rural fringe), and self-rated family economic status (poor, moderate, and good).

Clinical characteristics included clinical setting (outpatient vs. inpatient), the presence of two major medical conditions (hypertension and diabetes mellitus), cataract subtype (cortical, nuclear, posterior subcapsular, and mixed), affected site (unilateral vs. bilateral), and treatment stage (before vs. after surgery). This clinical information was collected by a review of medical records.

A checklist was used to collect data on patients' major medical conditions, which included 13 specific physical illnesses: hypertension, diabetes, heart disease, stroke and other cerebrovascular diseases, chronic obstructive pulmonary disease, cancer, tuberculosis, chronic prostatitis, chronic gastric ulcer, Parkinson's disease, anemia, hepatic sclerosis, and arthritis.

Depressive symptoms during the past week were assessed with depression subscale of the validated Chinese HADS, which has seven items and all are answered on a 0-3 scale (Zigmond \& Snaith 1983). The total score varies between 0 and 21, with higher scores representing more severe depressive symptoms. A cut-off score of nine or higher is recommended to denote clinically significant depressive symptoms in Chinese population (Yang et al. 2014). The strength of HADS is that it can avoid reliance on somatic symptoms (i.e., pain, fatigue and insomnia) for assessing depressive symptoms (Zigmond \& Snaith 1983). Given that many 
124 patients with cataract have chronic illnesses, this tool is particularly suitable for detecting

125 depression in cataract patients with satisfactory validity and specificity.

126 Statistical analysis

127 Prevalence rates of depressive symptoms in the total sample and different subsamples according 128 to socio-demographic and clinical characteristics were calculated. Comparisons of rates across 129 subsamples were made by using Chi-square test. Multiple logistic regression model with a 130 forward stepwise entry of significant variables in the above Chi-square test were used to identify 131 factors associated with depressive symptoms. Odds ratios (ORs) and 95\%CIs were used to 132 quantify associations between factors and depressive symptoms. Significance level was set at 1330.05 (two-sided) in all analyses. SPSS software version 12.0 was used to analyze the data.

134

135

136

137

138

139

140

141

142

143

144

145

146

147

148

149

150

151

152

154

\section{Results}

During the study period, 370 patients were eligible for the study and all were invited to participate. Finally, a total of 339 patients with cataract completed the survey (response rate: 91.6\%). Among the final sample, $47.5 \%$ were men and the average age was 67.1 years (range: 20-90, standard deviation: 10.9). Detailed socio-demographic and clinical characteristics of the study sample are displayed in Table 1.

Altogether, 81 patients were detected as having depressive symptoms. The corresponding prevalence of depressive symptoms was $23.9 \%$ (95\%CI: $19.4-28.4 \%$ ).

Results of comparisons across subsamples (Table 1) show that, patients having an educational attainment of primary school and below, having marital status of "others", residing in rural area, rating their family economic status as "poor", suffering from nuclear cataract, having bilateral cataracts, and being after surgery had significantly higher prevalence rates of depressive symptoms than their counterparts without these attributes $(P \leqslant 0.046)$.

In multiple logistic regression, depressive symptoms were significantly associated with an education level of primary school and below $(\mathrm{OR}=1.93, \mathrm{P}=0.038)$, marital status of "others" $(\mathrm{OR}=3.15, \mathrm{P}<0.001)$, poor family economic status $(\mathrm{OR}=2.26, \mathrm{P}=0.010)$, nuclear cataract $(\mathrm{OR}=4.32, \mathrm{P}<0.001)$, and mixed cataract $(\mathrm{OR}=2.76, \mathrm{P}=0.017)$ (Table 2).

\section{Discussion}

In recent years, the importance of depression in patients with cataract and other eye diseases has 
155 been increasingly recognized, but this mental health issue remains poorly detected and managed 156 in China's clinical practice in ophthalmology (Li et al. 2013). One of the most important reason 157 is that Chinese ophthalmologists are unaware of depression and its clinical characteristics among 158 their patients. To fill this gap, we investigated the clinical epidemiology of depressive symptoms 159 in patients with cataract. By using HADS, 23.9\% of the Chinese patients with cataract were 160 found to be depressed during the past week and the prevalence of depressive symptoms varied 161 across different socio-demographic and clinical subgroups. Factors significantly associated with 162 depressive symptoms in this patient population were an education level of primary school and 163 below, marital status of "others", poor family economic status, and two subtypes of cataract: 164 nuclear and mixed.

In China, large-scale population-based studies have shown that 5.9-8.3\% of the Chinese 166 general adults suffer from depressive symptoms (Lin et al. 2018; Tu et al. 2018; Zhou et al.

167 168 169 170 2014). Compared to these estimates in the general population, we found a much higher prevalence of depressive symptoms in Chinese patients with cataract, suggesting the high risk of depressive symptoms among patients with cataract. Compared to two previous studies that used HADS to assess the prevalence of depressive symptoms in Chinese patients with cataract (16.718.0\% ) (Chen et al. 2019; Zhang et al. 2018), this study also demonstrated higher prevalence in our sample. This difference could be ascribed to the sample characteristics, for example, the two studies recruited patients awaiting for surgery only, but we recruited both patients before and after surgery. The significantly higher prevalence of depressive symptoms in patients after than before surgery in our study (Table 1) supports this speculation.

In line with the higher risk of depressive disorders in Chinese older adults with an educational attainment of primary school and below in comparison to those with an education level of middle school and above (Zhong et al. 2020b), we found that an educational attainment of primary school and below was significantly associated with depressive symptoms in Chinese patients with cataract. This relationship may be attributed to the poor mental health literacy of patients with a low level of education, which limits their ability to maintain and promote mental health. In general, family support from spouses can buffer the negative effect of physical illnesses such as cataract on mental health, thereby reducing the risk of depression among patients having spouses (Ivbijaro et al. 2019; Zhong et al. 2020a). This may explain the significant association between marital status of "others" and depressive symptoms in patients with cataract. Consistent 
186

187

188

189

190

191

192

193

194

195

196

197

198

199

200

201

202

203

204

205

206

207

208

209

210

211

212

213

214

215

216

with the increased risk of depression in adults of a low socio-economic status (Villarreal-Zegarra \& Bernabe-Ortiz 2020; Zhong et al. 2015), poor family financial status was significantly associated with depressive symptoms in our study. In general, persons of a low socio-economic status may have inadequate social support resources to cope with their mental health issues, resulting in elevated risk of depression among these persons.

In patients with eye diseases, researchers have found the elevated risk of depression among those with severe visual impairment (Li et al. 2013). Because patients with nuclear and mixed cataracts are more likely to have poor vision, the significant associations of depressive symptoms with nuclear and mixed cataracts are expected in our study.

This study has a few limitations. First, this is an observational study so the correlates of depressive symptoms are not, strictly speaking, risk factors. Whether or not the identified correlates cause depressive symptoms need to be examined by prospective follow-up or even interventional studies. Second, our study only measured depressive symptoms, not depressive disorders; so it is not known how many of these patients were sufficiently impaired to justify a clinical diagnosis and psychiatric treatment. Third, utilization of mental health services of patients with cataract is also essential for the development and planning of health services in clinical practice in ophthalmology, but we did not collect these data. Fourth, in general, Chinese older adults are less likely to report their depressive feelings, particularly when HADS was administered in a face-to-face interview, as what we have done in this study. This may result in an underestimation of the prevalence of depressive symptoms. Finally, some other potential factors associated with depression (smoking, exercise habit, lack of social support, length of illness, functional imperilments, failure in cataract surgery, etc.) were not assessed in the study so it remains unknown whether or not these factors would also be associated with depressive symptoms in patients with cataract.

\section{Conclusions}

In summary, depressive symptoms are common among Chinese patients with cataract, indicating the high risk of depression in this patient population. Given the many negative impacts of depression to patients and our society, there is a pressing need to identify and address depression and other mental health problems of Chinese patients with cataract. Among patients with cataract, depressive symptoms are associated with education, marital status, economic status, and 
217 subtype of cataract. Efforts to prevent or reduce depression in clinical practice in ophthalmology 218 may be effective to target on those who are poorly educated, are not married, have poor family 219 economic status, and suffer from nuclear and mixed cataracts. Services for patients with cataract 220 in clinical practice should include regular screenings for those at risk for depression and other 221 mental health problems, expanded psychosocial supports, and, when necessary, psychiatric 222 assessment and treatment.

223

224

225

226

227

228

229

230

231

232

233

234

235

236

237

238

239

240

241

242

243

244

245

246

247

248

\section{Acknowledgements}

The authors thank all the research staff for their team collaboration work and all the primary care physicians and older adults involved in this study for their cooperation and support.

\section{References}

Chen W, Chen G, Zheng J, and Wang L. 2019. Comparison of uncertainty in illness, anxiety and depression in patients with glaucoma versus patients with cataract and their related influencing factors. Acad J Chin PLA Med Sch 40:1160-1164. (in Chinese)

Chen X. 2010. The research of senile cataract patients' depression situation before and after surgery. China Modern Med 17:132-133. (in Chinese)

Fraser ML, Meuleners LB, Ng JQ, and Morlet N. 2013. Driver self-regulation and depressive symptoms in cataract patients awaiting surgery: a cross-sectional study. BMC Ophthalmol 13:45.

Freeman EE, Gresset J, Djafari F, Aubin MJ, Couture S, Bruen R, Laporte A, and Boisjoly H. 2009. Cataract-related vision loss and depression in a cohort of patients awaiting cataract surgery. Can J Ophthalmol 44:171-176.

He J, and Gao W. 2015. The occurence of depression and its association with chronic diseases among community-dewlling older adults with cataract in Wuhan. Chin J Gerontol 35:58805882. (in Chinese)

Ivbijaro G, Kolkiewicz L, Goldberg D, Riba MB, N'jie INS, Geller J, Kallivayalil R, Javed A, Švab I, Summergrad P, Laher S, and Enum Y. 2019. Preventing Suicide, Promoting Resilience: Is This Achievable From a Global Perspective? Asia Pac Psychiatry 11:e12371.

Li W, Zhong B, Liu X, Huang X, Dai X, Hu Q, Zhang H, and Xu H. 2013. Depressive symptoms among the visually disabled in Wuhan: an epidemiological survey. Shanghai Arch Psychiatry 25:306-313. 
249 Lin X, Yw W, Ye Y, and Zhong W. 2018. The status of depression and its influencing factors in

250

251

252

253

254

255

256

257

258

259

260

261

262

263

264

265

266

267

268

269

270

271

272

273

274

275

276

277

278

279 adults in Fujian province. Chin J Health Statistics 35:850-854. (in Chinese)

Meuleners LB, Hendrie D, Fraser ML, Ng JQ, and Morlet N. 2013. The impact of first eye cataract surgery on mental health contacts for depression and/or anxiety: a population-based study using linked data. Acta Ophthalmol 91:e445-449.

Mitsonis CI, Mitropoulos PA, Dimopoulos NP, Mitsonis MI, Andriotis NM, Gitsa OE, and Mitsonis IM. 2006. Anxiety and depression in cataract surgery: a pilot study in the elderly. Psychol Rep 99:257-265.

Mylona I, Floros G, Dermenoudi M, Ziakas N, and Tsinopoulos I. 2020. A comparative study of depressive symptomatology among cataract and age-related macular degeneration patients with impaired vision. Psychol Health Med:1-7.

Palagyi A, Rogers K, Meuleners L, McCluskey P, White A, Ng JQ, Morlet N, and Keay L. 2016. Depressive symptoms in older adults awaiting cataract surgery. Clin Exp Ophthalmol 44:789-796.

Pellegrini M, Bernabei F, Schiavi C, and Giannaccare G. 2020. Impact of cataract surgery on depression and cognitive function: a systematic review and meta-analysis. Clin Exp Ophthalmol. doi: 10.1111/ceo.13754.

Tang Y, Wang X, Wang J, Huang W, Gao Y, Luo Y, Yang J, and Lu Y. 2016. Prevalence of AgeRelated Cataract and Cataract Surgery in a Chinese Adult Population: The Taizhou Eye Study. Invest Ophthalmol Vis Sci 57:1193-1200.

Tian F, Ren B, He Y, Jia J, Liu H, and Pei J. 2014. An epidemiological survey of cataract mong adults aged 50 years and above in rural, Shaanxi Province. Int Eye Sci 14:629-632.

Tu Q, Han A, Qin Y, Lin P, and Xiang Y. 2018. Prevalence of depression in adult residents and its influence factors in Jiangsu province. Modern Med J 46:1205-1209. (in Chinese)

Villarreal-Zegarra D, and Bernabe-Ortiz A. 2020. Association Between Arterial Hypertension and Depressive Symptoms: Results From Population-Based Surveys in Peru. Asia Pac Psychiatry. doi: 10.1111/appy.12385.

Wang H, Sun HP, Wang P, Xu Y, and Pan CW. 2016. Cataract and Depressive Symptoms among Older Chinese Adults. Optom Vis Sci 93:1479-1484.

Yang Y, Ding R, Hu D, Zhang F, and Sheng L. 2014. Reliability and validity of a Chinese version of the HADS for screening depression and anxiety in psycho-cardiological outpatients. 
280

281

282

283

284

285

286

287

288

289

290

291

292

293

294

295

296

297

298

299

Compr Psychiatry 55:215-220.

Yuan Z, and Li L. 2016. A review on epidemiological studies of blindness and vision impariments in China. Medicine and Pharmacy of Yunnan 37:345-349. (in Chinese)

Zhang D, Fan Z, Gao X, Huang W, Yang Q, Li Z, Lin M, Xiao H, and Ge J. 2018. Illness uncertainty, anxiety and depression in Chinese patients with glaucoma or cataract. Sci Rep $8: 11671$.

Zhong BL, Liu TB, Chan SS, Jin D, Hu CY, Dai J, and Chiu HF. 2015. Prevalence and correlates of major depressive disorder among rural-to-urban migrant workers in Shenzhen, China. J Affect Disord 183:1-9.

Zhong BL, Luo W, Xu YM, Li WX, Chen WC, and Liu LF. 2020a. Major depressive disorder in Chinese persons with speech disability: High rates of prevalence and perceived need for mental health care but extremely low rate of use of mental health services. J Affect Disord 263:25-30.

Zhong BL, Ruan YF, Xu YM, Chen WC, and Liu LF. 2020b. Prevalence and recognition of depressive disorders among Chinese older adults receiving primary care: A multi-center cross-sectional study. J Affect Disord 260:26-31.

Zhou X, Bi B, Zheng L, Li Z, Yang H, Song H, and Sun Y. 2014. The prevalence and risk factors for depression symptoms in a rural Chinese sample population. PLoS One 9:e99692.

Zigmond AS, and Snaith RP. 1983. The hospital anxiety and depression scale. Acta Psychiatr Scand 67:361-370. 


\section{Table $\mathbf{1}$ (on next page)}

Socio-demographic and clinical characteristics of patients with cataract and rates of depressive symptoms according to socio-demographic and clinical variables.

Columns 1-3 are socio-demographic and clinical variables, and column 5 is prevalence of depressive symptoms. 
1 Table 1. Socio-demographic and clinical characteristics of patients with cataract and rates of depressive symptoms according to socio-demographic and clinical variables

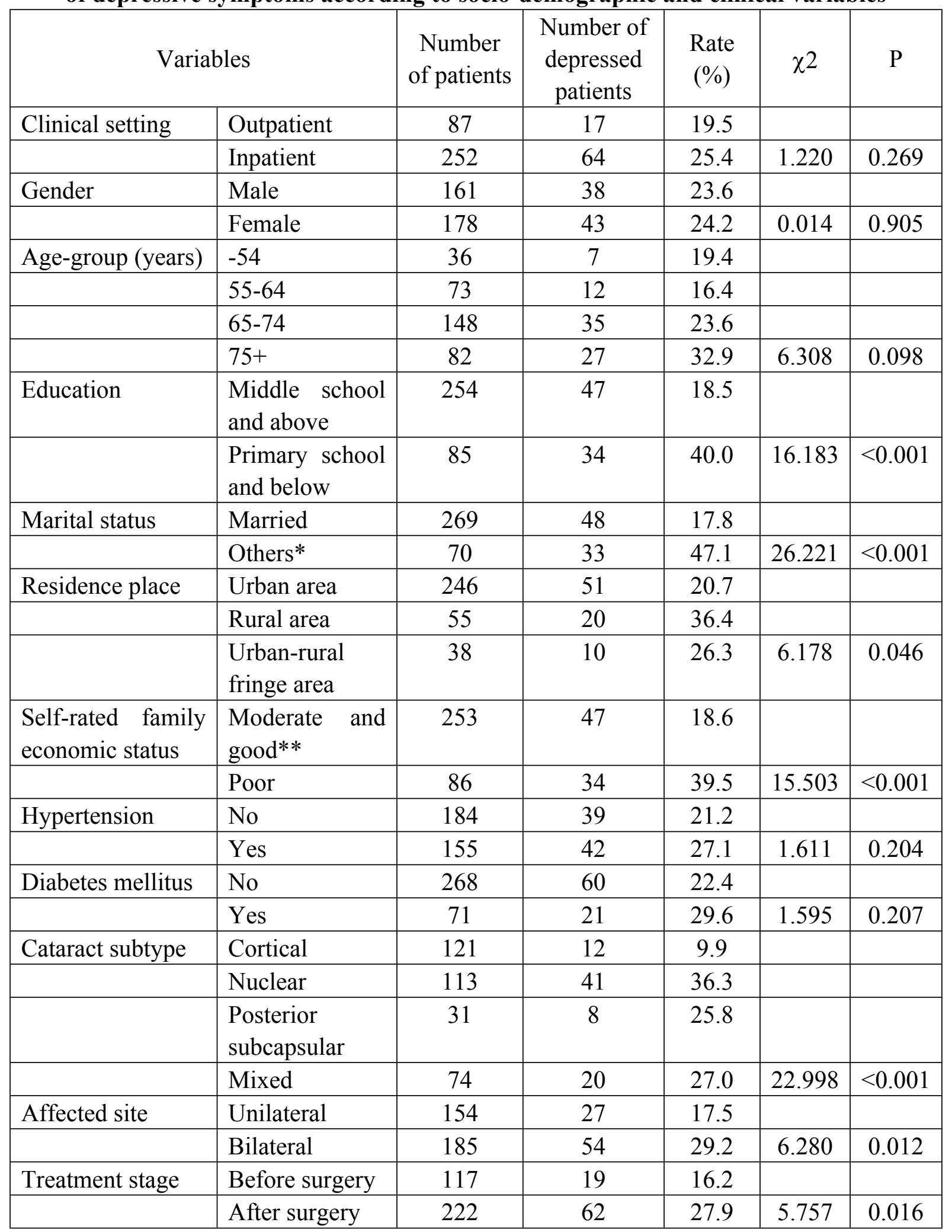

* "Others" includes never-married, remarried, cohabitating, separated/divorced, and widowed. 
$4 * *$ Only 9 patients rated their family economic status as "good", so "moderate" and "good" were 5 classified as one category. 


\section{Table 2 (on next page)}

Results of multiple logistic regression on factors significantly associted with depressive symptoms in Chinese patients with cataract.

Columns 1-2 are socio-demographic and clinical variables and column 3 is OR $(95 \% \mathrm{Cl})$ of these variables. 
1

2

Table 2. Results of multiple logistic regression on factors significantly associted with depressive symptoms in Chinese patients with cataract

\begin{tabular}{|l|l|c|c|c|c|c|}
\hline \multicolumn{2}{|c|}{ Variables } & $\begin{array}{c}\text { Coefficien } \\
\mathrm{t}\end{array}$ & $\begin{array}{c}\text { Standard } \\
\mathrm{error}\end{array}$ & $\begin{array}{c}\text { Wald } \\
\chi 2\end{array}$ & $\begin{array}{c}\text { OR } \\
(95 \% \mathrm{CI})\end{array}$ & $\mathrm{P}$ \\
\hline Education & $\begin{array}{l}\text { Middle school } \\
\text { and above }\end{array}$ & 1 & & & 1 & \\
\hline & $\begin{array}{l}\text { Primary } \\
\text { school and } \\
\text { below }\end{array}$ & 0.656 & 0.315 & 4.326 & $\begin{array}{c}1.93(1.04, \\
3.58)\end{array}$ & 0.038 \\
\hline Marital status & Married & 1 & & & 1 & \\
\hline & Others* & 1.146 & 0.308 & 13.836 & $\begin{array}{c}3.15(1.72, \\
5.75)\end{array}$ & $<0.001$ \\
\hline $\begin{array}{l}\text { Self-rated family } \\
\text { economic status }\end{array}$ & $\begin{array}{l}\text { Moderate and } \\
\text { good** }\end{array}$ & 1 & & & 1 & \\
\hline & Poor & 0.814 & 0.317 & 6.618 & $\begin{array}{c}2.26(1.21, \\
4.20)\end{array}$ & 0.010 \\
\hline $\begin{array}{l}\text { Subtype } \\
\text { cataract }\end{array}$ & Cortical & 1 & & & 1 & \\
\hline & Nuclear & 1.463 & 0.379 & 14.884 & $\begin{array}{c}4.32(2.05, \\
9.08)\end{array}$ & $<0.001$ \\
\hline & Mixed & 1.017 & 0.425 & 5.726 & $\begin{array}{c}2.76(1.20, \\
6.36)\end{array}$ & 0.017 \\
\hline
\end{tabular}

* "Others" includes never-married, remarried, cohabitating, separated/divorced, and widowed. 